\title{
Cirugía de preservación valvular como alternativa en enfermedad de la raíz aórtica. Resultados alejados
}

\author{
Aortic Valve Sparing Surgery as Alternative in Aortic Root Disease. Long-term Outcomes \\ FERNANDO PICCININI ${ }^{\circ}$, ADRIANA ARANDA ${ }^{2}$, JUAN MARIANO VRANCIC ${ }^{1 \oplus}$, MARIANO CAMPORROTONDO $^{1} \odot$, \\ JUAN CARLOS ESPINOZA ${ }^{1 \oplus,}$ LEONARDO SEOANE$^{3} \oplus$, DANIEL NAVIA $^{1 \oplus}$
}

\begin{abstract}
RESUMEN
Introducción: Desde 1968, la enfermedad aneurismática de la raíz aórtica ha sido tratada mediante el remplazo con tubo valvulado. En las últimas décadas la cirugía de preservación valvular surgió y evolucionó como una opción al remplazo protésico. Objetivo: Reportar la experiencia institucional en la técnica de preservación valvular y sus resultados a largo plazo.

Material y métodos: Revisión de 116 casos consecutivos con criterios de reparabilidad, intervenidos entre 2005 y 2019 . Previo ecocardiograma transesofágico (ETE) y angiotomografía (AngioTC), se procedió quirúrgicamente acorde a la clasificación anatomofuncional, con la combinación de técnicas. Se realizó control intraoperatorio y conversión a remplazo según el criterio del cirujano interviniente. Se reportan las variables intraoperatorias, la morbimortalidad intrahospitalaria y la mortalidad, la libertad de insuficiencia valvular significativa y la reoperación en el seguimiento clínico y ecocardiográfico.

Resultados: La edad media era $56 \pm 15,6$ años, varones 73\%, 59\% asintomáticos, intervenidos por diámetro aórtico (52 $\pm 11,7$ $\mathrm{mm}$ ) o progresión de valvulopatía. En el posprocedimiento, $4 \%$ de los casos resultó con insuficiencia leve o nula y 2 conversiones (1,7\%); mortalidad hospitalaria 0,9\%. A 10 años de seguimiento, sobrevida actuarial del $88 \%$ y libertad de insuficiencia significativa (moderada/grave) 79\%. Se reintervinieron 5 casos, a un intervalo promedio de 9,1 años, libertad de reoperación de $90 \%$ a 10 años. No se registraron eventos tromboembólicos ni hemorrágicos mayores.

Conclusión: las técnicas de preservación valvular aórtica, en contexto de enfermedad de la raíz, resultan una opción factible, segura y estable en el tiempo.
\end{abstract}

Palabras clave: Válvula aórtica - Aneurisma - Preservación valvular

\begin{abstract}
Background: Since 1968, ascending aorta replacement with a valved conduit has been the standard practice for aortic root aneurysm. By the end of the 20th century, aortic valve sparing operation emerged and evolved as an alternative to aortic valve replacement.

Objective: The aim of this study was to report our experience with aortic valve sparing technique and its long -term outcomes. Methods: A total of 116 consecutive cases with criteria of repairability operated on between 2005 and 2019 were analyzed. Preopera- tive transesophageal echocardiography (TEE) and computed tomography angiography (CTA) were used in combination to determine the aortic phenotype based on a previous anatomical and functional classification. Perioperative control was performed and conver- sion to aortic valve replacement was left to the discretion of the attending surgeon. Intraoperative variables, in-hospital morbidity and mortality, freedom from significant aortic regurgitation (AR) and reoperation in the clinical and echocardiographic follow-up were reported.

Results: Mean age was $56 \pm 15.6$ years and $73 \%$ were men; $59 \%$ were asymptomatic, and the reason for the intervention was the aortic diameter $(52 \pm 11.7 \mathrm{~mm})$ or progression of AR. After the procedure, $4 \%$ of the cases presented mild or trivial AR and 2 patients required conversion to aortic valve replacement (1.7\%). In hospital mortality was $0.9 \%$. Actuarial survival was $88 \%$ at 10 years, and 79\% were free from significant (moderate/severe) AR. Five cases underwent reoperation after a mean interval of 9.1 years and free- dom from reoperation at 10 years was $90 \%$. There were no major thromboembolic or bleeding events. Conclusion: Aortic valve sparing technique in the setting of aortic root disease is a feasible and safe option, and stable over time.
\end{abstract}

Key words: Aortic valve - Aneurysmal disease - Valve sparing procedure

\section{INTRODUCCIÓN}

Desde las publicaciones de Bentall en 1968, el remplazo de aorta ascendente y válvula aórtica mediante la inserción de un tubo valvulado, biológico o mecánico, constituye el tratamiento estándar de la enfermedad aneurismática de la raíz, independientemente del grado de enfermedad valvular. (1) Esa técnica constituye

Rev ARgent CARDiol 2021;89:115-123. http://dx.doi.org/10.7775/rac.es.v89.i2.18571

Recibido: 01/09/2020 - Aceptado: 19/12/2020

Dirección para separatas: Dr. Fernando Piccinini - Blanco Encalada 1543 - (1428) - CABA - Tel.: 54-11-4787 7502 - E-mail:fpiccinini@icba.com.ar 
un recurso válido, factible y reproducible en casos de estenosis con compromiso de raíz o aorta proximal; sin embargo, en asociación con regurgitación pura, la presencia de una válvula disfuncional, pero estructuralmente normal, pone en duda la necesidad del remplazo protésico y sus implicancias, sean la anticoagulación requerida para prótesis mecánicas, el deterioro estructural y trombosis subclínica de las bioprótesis, o el riesgo de endocarditis para ambos modelos.

En la década de 1980, Yacoub (2) introdujo el concepto de remodelación, y una década más tarde, David y Feindel publicaron su serie de reimplante valvular. (3) Desde entonces, cientos de publicaciones atestiguan la evolución de la técnica, su clasificación fisiopatológica, las condiciones anatómicas óptimas para su aplicación, la selección de pacientes y los métodos de evaluación pre y posprocedimiento, que alcanzaron resultados comparables e incluso, superiores al remplazo convencional en términos de morbimortalidad. En similitud con las plásticas mitrales, de demostradas ventajas, la aplicación y la expansión de la reparación aórtica se ve limitada por la exigencia técnica y el riesgo sustancial de falla, sumados a la escasa información sobre factibilidad y resultados en nuestro medio.

En 2005, nuestra institución inició un programa de preservación valvular que ha evolucionado al punto de constituir la primera elección en pacientes con enfermedad aneurismática, en ausencia de degeneración o estenosis valvular. El objetivo de esta presentación es revisar la clasificación fisiopatológica y los criterios de selección de los pacientes, así como reportar los resultados a corto y largo plazo, en términos de recurrencia de insuficiencia valvular y reintervención, a fin de promover su utilización, extensamente recomendada, pero escasamente aplicada. $(4,5)$

\section{MATERIAL Y MÉTODOS}

\section{Población en estudio}

Entre enero de 2005 y diciembre de 2019, fueron derivados 712 pacientes a nuestro departamento con diagnóstico de enfermedad aneurismática de la aorta ascendente e indicación quirúrgica, por guías clínicas. $(6,7)$ Excluídos aquellos con estenosis de grado significativo, disecciones agudas, endocarditis activa, reoperación o con criterios ecocardiográficos de no reparabilidad (8), $116 \mathrm{P}$ fueron consecutivamente incluídos en un programa de reparación valvular, de seguimiento anual por protocolo. Todos fueron previamente informados de la posibilidad de reparación, a la que consintieron.

\section{Data clínica preoperatoria}

Previo a la intervención se realizaron una ecografía transtorácica, ETE y tomografía multicorte de aorta torácica. Los estudios ecocardiográficos fueron realizados con equipo Philips iE33 con transductor $\mathrm{S} 5-1 \mathrm{MHz}$ y sonda multiplanar $\mathrm{X} 7-2 \mathrm{~T} \mathrm{MHz}$ y/o Philips EpiQ 7 con transductor x Matrix 2D/3D y sonda transesofágica 3D de $7 \mathrm{MHz}$.

Por medio de un examen ecotranstorácico se evaluó la presencia y el grado de insuficiencia aórtica (IAO), diámetros ventriculares, función sistólica y medidas de la raíz aórtica, siguiendo recomendaciones de las Asociaciones Americana y
Europea de Cardiología (sin insuficiencia, leve, moderada y/o grave).(9)· Por ETE se obtuvieron imágenes en eje corto y eje largo, entre 0 y 180 grados. Se evaluó el número de cúspides y en bicúspide, la presencia y las características del rafe y la simetría. La movilidad valvar fue reportada como normal, excesiva o restrictiva, considerando prolapso a toda eversión o abombamiento del borde libre hacia el tracto de salida, por debajo del plano anular, en diástole; se determinó la presencia y el tipo de fibrosis o calcificación valvular. (8)

Con Doppler color se evaluó la dirección del jet regurgitante. En el plano sagital se midieron los diámetros del anillo, los intersenos, la unión sinotubular (UST), y tubular ascendente (en modo 2D y 3D, con reconstrucción multiplanar), determinando el fenotipo del aneurisma. (9).

La AngioTC fue realizada con gatillado cardíaco y adquisición de tres tiempos, con tiempo arterial al 75\% de fase cardíaca. Perpendicular al eje mayor del vaso se determinó el diámetro máximo en el nivel del anillo, el interseno, la UST y tubular, así como la configuración valvular, la geometría anular, la disposición de vasos supraaórticos y enfermedad coronaria. En casos no concluyentes, se realizó cinecoronariografía. Con el objetivo de facilitar la selección de técnica quirúrgica, se utilizó la clasificación anatomofuncional modificada por El Khoury (Figura 1) para fenotipo aórtico. (10)

\section{Procedimiento quirúrgico}

Todas las intervenciones fueron realizadas por esternotomía mediana y circulación extracorpórea por canulación directa del arco aórtico o axilar derecha, dependiendo de la extensión distal del aneurisma. Se utilizó cardioplejía sanguínea intermitente y venteo pulmonar. Mediante aortotomía transversa alta, se procedió a la inspección valvular, y se observó el número y la disposición de las valvas, las fenestraciones, la ubicación y la extensión del rafe, fibrosis o calcificación, y la altura geométrica de las valvas determinantes de la factibilidad de reparación, así como el diámetro anular e interseno, y las características de la pared aórtica, independientemente de su diámetro.

Como se expresó, la selección de la técnica se ajustó a la clasificación anatomofuncional; los casos con dilatación por encima de la UST (subtipo 1A) fueron tratados mediante remplazo aorto-aórtico; los subtipos $1 \mathrm{~B}$ y $1 \mathrm{C}$, mediante técnica de reimplante o remodelado, inicialmente según la decisión del cirujano, y en la actualidad ligado al diámetro anular (remodelado con anillo $<25 \mathrm{~mm}$, reimplante si $\geq 25$ $\mathrm{mm}$ ), establecido por eco o mediante el uso de medidores intraoperatorios.

Excepcionalmente, la restricción anular se logró mediante anuloplastia externa con PTFE 2.0 o banda de teflon (Figura 1). Las lesiones valvares prediagnosticadas u observadas en la reparación, como prolapso (tipo 2), fueron tratadas por plicatura o resección del borde libre, a fin de ajustar su altura geométrica a 8-10 mm. Las restricciones, características de las bicúspides, se trataron con resección del rafe y cierre primario o plicatura con puntos separados de polipropilene 6.0 , dependiendo de su extensión y la altura de implante a nivel de la comisura frustra.

Finalizada la reparación, fueron evaluados con equipos Philips EPIQ y Sonosite Turbo con sonda transesofágica multiplanar 8-3 MHz. Se investigó, tanto la presencia y el grado de insuficiencia residual como el mecanismo responsable, con especial atención sobre la superficie y la altura de coaptación valvar, el prolapso residual y la dirección del jet regurgitante, así como el área y el gradiente transvalvular. La conversión a remplazo fue decisión del médico interviniente, conforme al resultado ecocardiográfico, la edad, el estado clínico y las comorbilidades del paciente. 


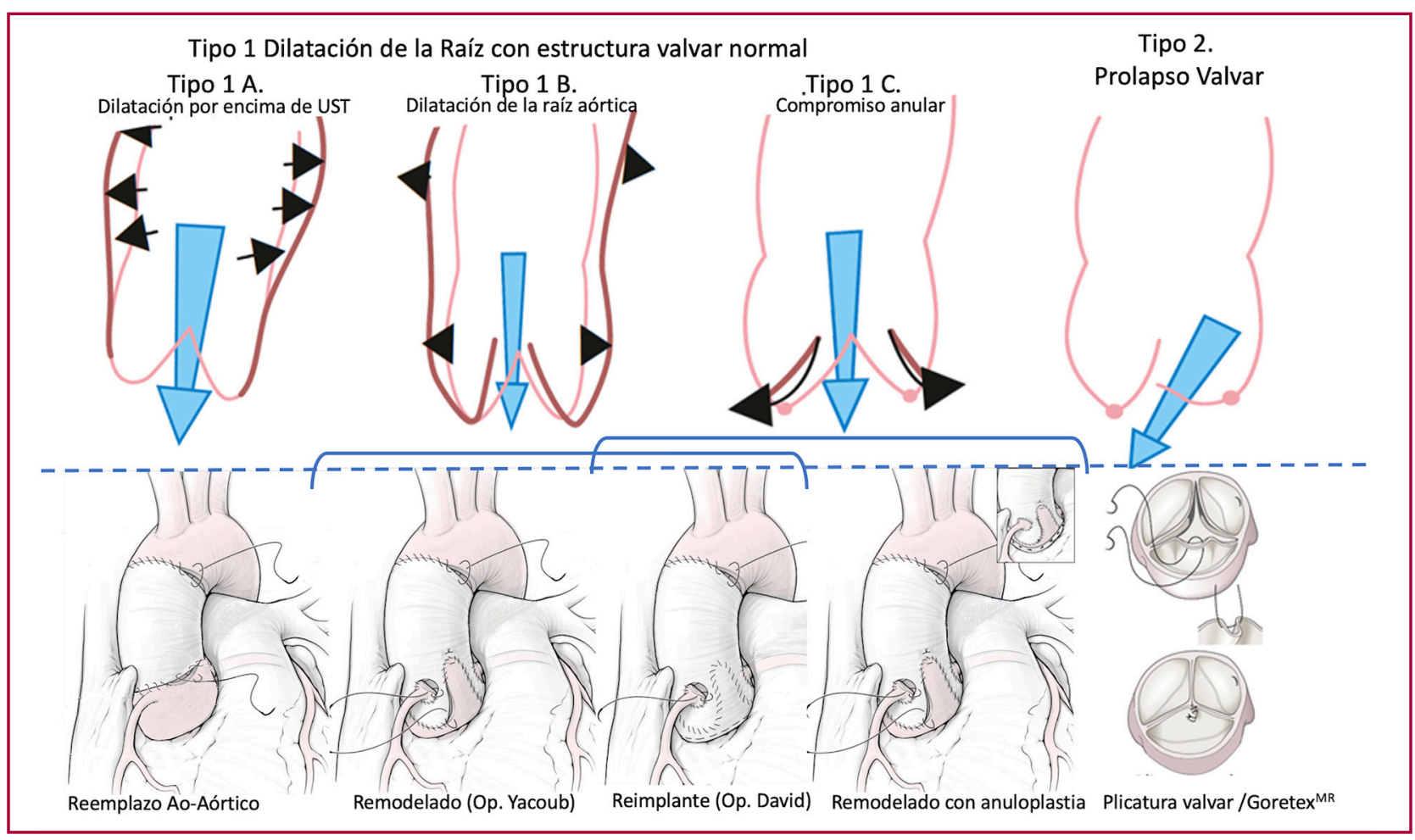

UST: unión sinotubular

Fig. 1. Arriba: Clasificación funcional modificada de El Khoury y col.(10) (excluidos tipo 1C, perforación y tipo 3, restrictiva). Abajo: Técnica quirúrgica utilizada. (Las lesiones tipo 1 y 2 pueden asociarse, resultando en técnicas quirúrgicas complementarias).

\section{Seguimiento}

La morbimortalidad perioperatoria se determinó siguiendo definiciones convencionales (Apéndice 1). Todos los pacientes fueron evaluados por ecocardiografía al alta, y seguidos por la clínica de valvulopatías; esa evaluación clínica y ecocardiográfica fue repetida a los 3, 6 y 12 meses y, anualmente, asociando AngioTC. Ante sospecha clínica de endocarditis, aumento de gradientes o progresión de IAO, se indicó ETE. En la evaluación de curvas de sobrevida y reincidencia de insuficiencia significativa, se consideró la variante valvular (bicúspide o tricúspide), asociada al resultado dispar en publicaciones previas.

\section{Análisis estadístico}

Las variables de tipo cuantitativas fueron expresadas como media \pm desvío estándar o mediana (rango intercuartilo) en los casos con distribución no gaussiana. Las variables cualitativas se reportaron como porcentaje. Las curvas de sobrevida libre de eventos se construyeron por el método de Kaplan-Meier. Las diferencias de supervivencia entre grupos se analizaron mediante log rank test. Para identificar predictores de mortalidad tardía significativos se realizó un análisis univariado y multivariado con la prueba de riesgos proporcionales de Cox. Las variables clínicas detalladas en la Tabla 1 se utilizaron para el análisis univariado, incluyendo en el modelo multivariado aquellas con valor de $\mathrm{p}<0,2$.

\section{Consideraciones éticas}

El estudio fue conducido de acuerdo con las normas nacionales e internacionales de investigación en seres humanos (Declaración de Helsinki y otras). La confidencialidad de los datos personales de los pacientes fue tratada de acuerdo con la ley 25326 de Protección de Datos Personales. Los pacientes fueron identificados mediante un código numérico, y sus datos filiatorios se mantienen en forma anónima.

\section{RESULTADOS}

La mediana de edad fue de $56 \pm 15,6$ años (rango 1878 ), $73 \%$ de sexo masculino, superficie corporal 1,99 \pm $0,2 \mathrm{~m}^{2}$. Las características basales se muestran en la Tabla 1. La principal indicación de intervención fue el diámetro aórtico indexado, su progresión o antecedentes heredofamiliares. Un escaso 10,3\% fue categorizado como síndrome de Marfan o Loeys Dietz.

\section{Procedimiento quirúrgico y resultados de la reparación}

Acorde al fenotipo aórtico, 17 casos (15\%) fueron sometidos a remplazo aorto aórtico (subtipo 1A), 16 casos (14\%) a remodelado, y los restantes 83 casos (71\%) a reimplante (subtipos 1B-1C).

En el grupo remodelado 2 casos $(12,5 \%)$ recibieron anuloplastia externa adicional. La elección de plicar la unión ventrículo-aórtica -sea por reimplante o anuloplastía externa- fue decisión del cirujano, pero basada en mediciones previas (media de anillo de $26 \pm 3,3 \mathrm{~mm}$ global, $23 \pm 3,6 \mathrm{~mm}$ para el grupo remodelado vs. 27 $\pm 4,5 \mathrm{~mm}$ para el grupo reimplante). La asociación de 


\section{Tabla 1. Variables preoperatorias global}

\begin{tabular}{|c|c|}
\hline Factores de riesgo & $\% / n$ \\
\hline Hipertensión & $61,8(71)$ \\
\hline Dislipemia & $30,7(35)$ \\
\hline Tabaquismo & $39,6(46)$ \\
\hline Diabetes & $1,7(2)$ \\
\hline Antecedentes, heredofamiliares & $22,4(26)$ \\
\hline Antecedentes patológicos $(n=116)$ & $\% / n$ \\
\hline Accidente cerebrovascular previo & $2,5(3)$ \\
\hline Enfermedad pulmonar moderada/grave & $6,5(8)$ \\
\hline Insuficiencia renal previa & $6,8(8)$ \\
\hline Infarto de miocardio previo & $0,8(1)$ \\
\hline Angioplastía previa & $3,4(4)$ \\
\hline Enfermedad coronaria significativa & $9,4(11)$ \\
\hline Cuadro clínico predominante & $\% / n$ \\
\hline Asintomáticos & $59(66)$ \\
\hline Disnea progresiva & $30(35)$ \\
\hline Angina inestable & $6(7)$ \\
\hline Insuficiencia cardíaca & $0,8(1)$ \\
\hline Síncope & $3,4(4)$ \\
\hline \multicolumn{2}{|l|}{ Ecocardiografía } \\
\hline Diámetro diastólico VI (mm) (med/DE) & $55 \pm 9,1$ \\
\hline Diámetro sistólico VI (mm) (med/DE) & $34 \pm 9,1$ \\
\hline Función ventricular Mod/Grave & $9,3(35)$ \\
\hline Configuración bicúspide & $21,6(35)$ \\
\hline Insuficiencia aórtica preop & 75,8 \\
\hline - Grado 0 & $6(7)$ \\
\hline - Grado 1 & $19,8(23)$ \\
\hline - Grado 2 o mayor & $74,2(86)$ \\
\hline Fibrosis - calcificación Grado 2/3 & $5,1(6)$ \\
\hline Prolapso valvar (\%) & $18,1(21)$ \\
\hline Tomografía axial computada & (med/DE) \\
\hline Diámetro anular aórtico, (mm) & $26 \pm 4,5$ \\
\hline Diámetro aórtico sinusal, (mm) & $49,5 \pm 7,8$ \\
\hline Diámetro UST, (mm) & $52 \pm 11,7$ \\
\hline Diámetro tabular aórtico (mm) & $42 \pm 8$ \\
\hline
\end{tabular}

med/DE: media/ desvío estándar

UST: unión sinotubular

VI: ventrículo izquierdo

lesión tipo 2 (prolapso por eco o inspección directa) fue del $55 \%$, sin diferencia entre grupos. Su plicatura directa representó la variante técnica más utilizada, mayormente en el subgrupo de reimplante.
Otras variables técnicas se modificaron a lo largo de la serie, con un abandono precoz de la resuspensión con puntos subcomisurales o las suturas de Goretex ${ }^{\circledR}$ sobre bordes libres. Discontinuada la circulación extracorpórea, por ETE se reportó un $94 \%$ de pacientes sin IAO residual significativa, y 7 casos $(6 \%)$ de IAO de moderada a grave, con una tasa de conversión a remplazo del 1,7\% (2 casos). En los 5 casos restantes $(4,3 \%)$ se toleró insuficiencia residual moderada, en contexto etario, CEC prolongada y comorbilidad asociada, todos ellos intervenidos en los primeros 5 años del programa. Los datos intraoperatorios y los resultados están resumidos en la Tabla 2.

\section{Morbimortalidad periprocedimiento}

Las complicaciones periprocedimiento fueron de baja incidencia, y son reportadas en la Tabla 3.

\section{Seguimiento clínico y ecocardiográfico}

La mediana de seguimiento fue 4 años $(1,9-8,8)$, con una pérdida de 3 pacientes $(2,6 \%)$. Ocurrieron 8 muertes, 2 de origen cardíaco: una muerte súbita y un óbito por insuficiencia cardíaca secundaria a disfunción valvular. La sobrevida actuarial a 10 años fue $88 \%$, sin diferencias por fenotipo valvar (Figura 2A).

Se registraron 5 reoperaciones, todas ellas de carácter electivo: 3 casos (2,6\%), secundarias a endocarditis a 4 y 6 años, 1 caso por progresión de la IAO residual a 4,9 años, y el restante por estenosis, a 10,6 años. No se registraron complicaciones mayores o muertes en las reintervenciones. Un sexto paciente, con indicación por estenosis residual, rechazó la cirugía y representa un fallecido de causa cardíaca. La libertad de reintervención fue de $91 \%$ a 10 años (Figura 2B).

Por ecocardiografía se reportó reincidencia o progresión a IAO significativa en 12 pacientes $(10,4 \%)$ a 5,8 años promedio desde la intervención (Figura 2C). La libertad de IAO significativa fue de $79 \%$ a 10 años (Figura 2D). La regresión proporcional de Cox en análisis multivariado identificó al jet excéntrico como predictor independiente de progresión alejada (HR 17,6, IC 95\% 3,7-84,1, p <0,0001).

El 87\% de los pacientes demostró fracción de eyección del ventrículo izquierdo (FEVI) superior al 50\%, con mejoría de los diámetros ventriculares izquierdos (diámetro diastólico $54 \pm 9,1 \mathrm{~mm}$ pre a $49 \pm 6,5$ post; diámetro sistólico $34 \pm 9$ pre a $30 \pm 5,8$ post, $\mathrm{p} \leq 0,01$ ), estabilidad en gradientes residuales $(8 \pm 6,2 \mathrm{mmHg})$ y velocidad máxima transvalvular aórtica $(1,4 \pm 0,5 \mathrm{~m} / \mathrm{seg})$.

Clínicamente, el 92\% de los sobrevivientes (99/108) se reportó en CF I, independientemente del grado de IAO residual diagnosticada por imágenes. Se reportaron en el seguimiento 4 pacientes anticoagulados por remplazo valvular alejado mecánico, sin eventos hemorrágicos o tromboembólicos clínicos asociados al tratamiento. 
Tabla 2. Variables intraoperatorias globales y por técnica quirúrgica
Tabla 3. Complicaciones perioperatorias globales y por técnica quirúrgica

\begin{tabular}{|c|c|c|c|c|}
\hline Variable & $\begin{array}{c}\text { Global } \\
N=116 \\
\% /(n)\end{array}$ & $\begin{array}{c}\text { RAO-AO } \\
N=17 \\
\% /(n)\end{array}$ & $\begin{array}{c}\text { Remodelado } \\
N=16 \\
\% /(\mathbf{n})\end{array}$ & $\begin{array}{c}\text { Reimplante } \\
N=83 \\
\% /(n)\end{array}$ \\
\hline IAO moderada/grave preop. & $74,2(86)$ & $82,3(14)$ & $62,5(10)$ & $74,4(62)$ \\
\hline Configuración bicúspide & $21,5(25)$ & $23,5(4)$ & $31,2(5)$ & $19,2(16)$ \\
\hline Prolapso a la inspección & $27,5(32)$ & $29,4(5)$ & $12,5(2)$ & $30,1(25)$ \\
\hline Tto adicional valvas & $55(64)$ & $58,8(10)$ & $56,2(9)$ & $54,2(45)$ \\
\hline -Plicatura valvar & $28,4(33)$ & $11,8(2)$ & $25(4)$ & $32,5(27)$ \\
\hline -Resección valvar & $8,6(10)$ & $11,8(2)$ & $25(4)$ & $4,8(4)$ \\
\hline -Puntos subcomisurales & $18,9(22)$ & $52,9(9)$ & $25(4)$ & $10,7(9)$ \\
\hline -Sutura de PTFe & $3,4(4)$ & $5,9(1)$ & 0 & $3,6(3)$ \\
\hline Diámetro graft(mm)(moda/rango) & $30(24-34)$ & $28(26-34)$ & $38(26-30)$ & $30(24-30)$ \\
\hline Cirugía coronaria asociada & $10,3(12)$ & $29,4(5)$ & 0 & $8,4(7)$ \\
\hline Tiempo de clampeo (min)(med/DE & $153 \pm 48$ & $71 \pm 22$ & $142 \pm 44$ & $164 \pm 34$ \\
\hline Tiempos de CEC (min) (med/DE) & $178 \pm 52$ & $87 \pm 31$ & $162 \pm 47$ & $183 \pm 38$ \\
\hline Anuloplastía externa & $2,5(3)$ & $5,9(1)$ & $12,5(2)$ & 0 \\
\hline Sin insuficiencia residual & $45,7(53)$ & $35,2(6)$ & $37,5(6)$ & $49,4(41)$ \\
\hline IAO trivial - leve & $48,3(56)$ & $58,8(10)$ & $56,2(9)$ & $44,5(37)$ \\
\hline IAO moderada/grave & $6(7)$ & $5,8(1)$ & $6,2(1)$ & $6(5)$ \\
\hline Conversión/remplazo & $1,7(2)$ & 0 & $6(1)$ & $1,2(1)$ \\
\hline
\end{tabular}

RAO-AO: remplazo aorto aórtico. PTFe: preguntar. med/DE: media/desvío estándar. IAO: Insuficiencia aórtica. CEC: Circulación extracorpórea

\begin{tabular}{|lcccc|}
\hline Variable & $\begin{array}{c}\text { Global } \\
\mathbf{N}=116 \\
\% /(\mathbf{n})\end{array}$ & $\begin{array}{c}\text { RAO-AO } \\
\mathbf{N}=17 \\
\% /(\mathbf{n})\end{array}$ & $\begin{array}{c}\text { Remodelado } \\
\mathbf{N}=16 \\
\% /(\mathbf{n})\end{array}$ & $\begin{array}{c}\text { Reimplante } \\
\mathbf{N = 8 3} \\
\% /(\mathbf{n})\end{array}$ \\
\hline Sangrado médico & $20,7(24)$ & $\begin{array}{c}17,6(3) \\
25(4)\end{array}$ & $20,4(17)$ \\
\hline Reoperación por sangrado & $4,3(5)$ & 0 & 0 & $6,2(5)$ \\
\hline Fibrilación auricular & $22,4(26)$ & $17,6(3)$ & $37,5(6)$ & $20,4(17)$ \\
\hline Inotrópicos $>24 h$ & $24,1(28)$ & $23,5(4)$ & $6,25(1)$ & $27,7(23)$ \\
\hline ARM prolongada & $3,4(4)$ & $5,9(1)$ & $6,25(1)$ & $2,4(2)$ \\
\hline Marcapasos definitivo & $2,6(3)$ & 0 & 0 & $3,6(3)$ \\
\hline Insuficiencia renal & $6,03(7)$ & $11,7(2)$ & 0 & $6,02(5)$ \\
\hline Diálisis & $0,9(1)$ & 0 & 0 & $1,2(1)$ \\
\hline ACV con secuela & $0,9(1)$ & 0 & 0 & $1,2(1)$ \\
\hline Mortalidad & $0,9(1)$ & 0 & 0 & $1,2(1)$ \\
\hline Estadía hospitalaria días & $5 \pm 5.8$ & $5 \pm 3.8$ & $5 \pm 6.6$ & $5.5 \pm 2.8$ \\
(med/std - rango) & $(4-50)$ & $(4-19)$ & $(4-15)$ & $(5-50)$ \\
\hline
\end{tabular}

$\mathrm{med} / \mathrm{std}$ : mediana/desvío estandar

RAO-AO: remplazo aorto-aórtico. ARM: asistencia respiratoria mecánica. ACV: accidente cerebrovascular. med/DE: media/desvío estándar

\section{DISCUSIÓN}

Si bien múltiples publicaciones han mostrado excelentes resultados y estabilidad a largo plazo para la preservación aórtica (11-15), su aplicación real sigue siendo escasa, limitada a centros de alto volumen y experiencia en cirugía valvular. Representa el 19\% de las prácticas sobre aorta torácica según el registro de la Sociedad de Cirugía Torácica Americana (5), y un total de 7700 casos registrados en 5 años por el registro AVIATOR, con 55 centros europeos. Sus ventajas radican en la reducción de complicaciones asociadas al implante, especialmente anticoagulación y fenómenos embólicos, al margen de su perfil hemodinámico óptimo y estable en el tiempo. La dificultad técnica, la curva de aprendizaje y una cuestionada durabilidad son señaladas como debilidades por sus detractores, sumados a la probada eficacia y estandarización del procedimiento de Bentall. 
A

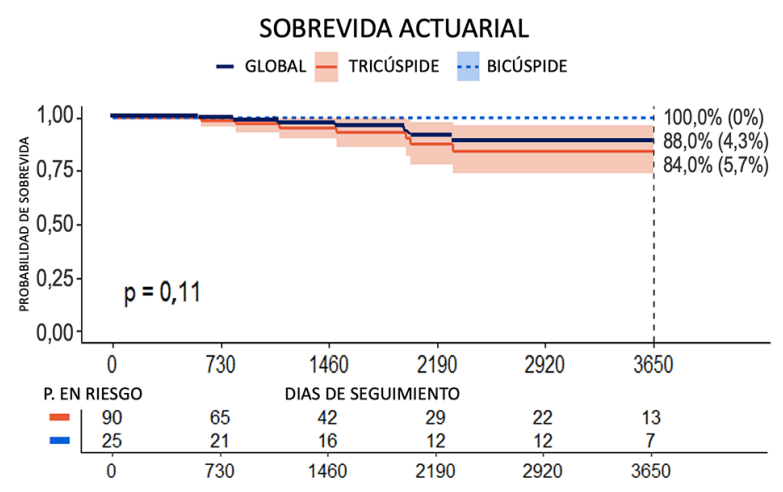

C

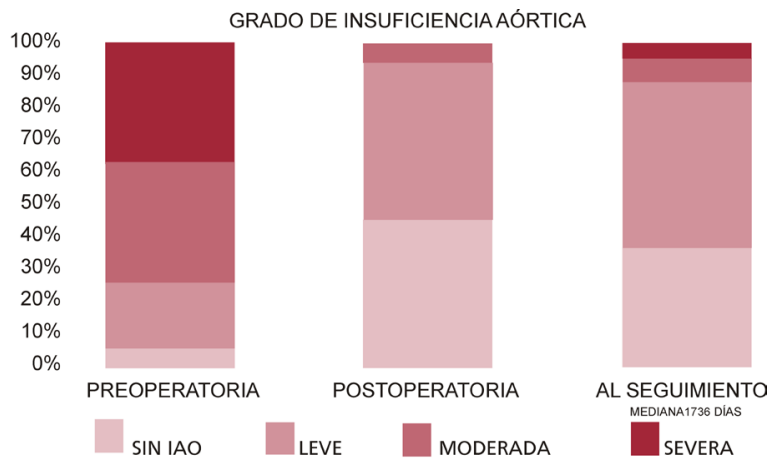

B

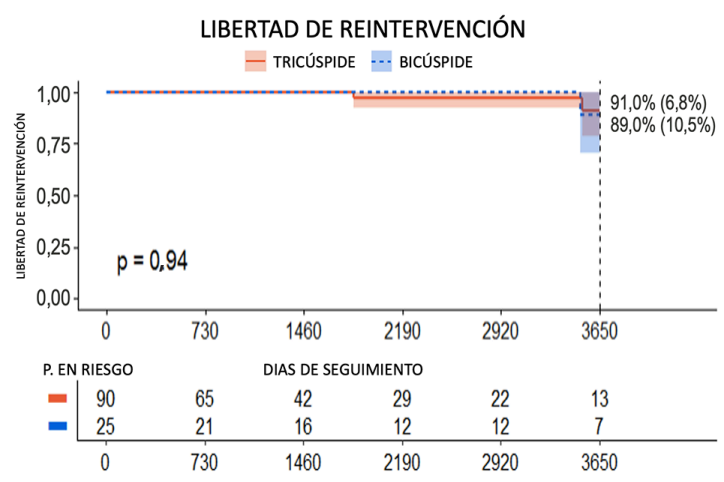

D

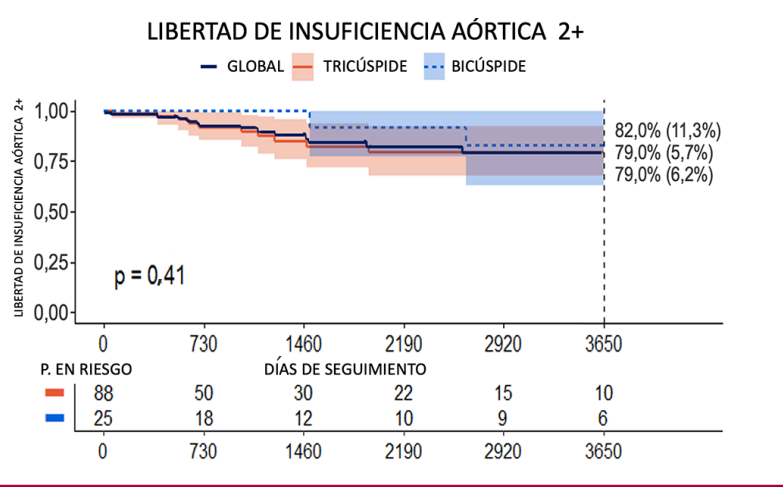

Fig. 2. A. Sobrevida actuarial (global y por fenotipo aórtico). B. Libertad de reintervención valvular (global y por fenotipo aórtico). C. Grado de IAO pre-operatoria, postop inmediata y a mediana de seg. D. Libertad de insuficiencia significativa (global y por fenotipo aórtico).

Diversos grupos han comparado las técnicas de preservación con el tradicional tubo valvulado, sea biológico o mecánico, en términos de morbimortalidad y libertad de reintervención, con resultados dispares. (16-18) Si bien no existen estudios prospectivos randomizados que comparen estrategias, un metaanálisis reciente de Elbatarny, (con 6218 pacientes en 26 publicaciones) establece una considerable ventaja de la preservación: $32 \%$ de reducción de mortalidad, $71 \%$ de reducción en eventos hemorrágicos y $64 \%$ en eventos tromboembólicos, a 5,8 $\pm 3,0$ años de seguimiento. (19)

Convencido de sus ventajas, nuestro grupo estableció una rutina de estudios ecocardiográficos preoperatorios, de cuyo análisis y valoración anatomofuncional depende el éxito de la preservación (7, 20-23). Emulando la plástica mitral, las lesiones tipo 3, restrictivas, tienen malos resultados alejados (7, 20); la altura efectiva de las valvas inferior a $16 \mathrm{~mm}$ en tricúspide y $19 \mathrm{~mm}$ en bicúspide, se asocia a resultados subóptimos. (23) Las correcciones valvares con parche de pericardio o material sintético, a excepción de mínimas fenestraciones, presentan una tasa de recurrencia precoz elevada, particularmente en pacientes con conformación bicúspide, siendo desaconsejadas $(24,25)$. Constituye esta la base para la exclusión de este tipo de lesiones en la siguiente serie. Asimismo, la presencia de marcada fibrosis o calcificación, especialmente a nivel del rafe en bicúspide, es un condicionante del éxito de la plástica y debe ser informada en la valoración prequirúrgica (26).

La correlación entre reporte ecocardiográfico y hallazgo intraoperatorio fue relativamente alta; se observaron variaciones en la detección de prolapso que no afectaron el resultado final. La definición del diámetro anular, sea ecocardiográfica o tomográfica, resulta esencial en la decisión de la técnica quirúrgica; los anillos superiores a $25 \mathrm{~mm}$, no tratados se asocian a recurrencia de IAO, por lo cual es primordial la estabilización anular, sea por anuloplastia externa o técnica de reimplante, consideración que justifica, en nuestra serie, la utilización de esta variable en la mayoría de los casos. (27-30)

Si bien no fue el objetivo de la serie comparar resultados en relación con el fenotipo aórtico, los pacientes con remplazo aorto-aórtico presentaron menor tasa de reincidencia de IAO significativa; ha sido probado que la regurgitación central (característica de este fenotipo) es estable en el seguimiento, en contraste con el reflujo excéntrico, identificado en nuestra serie como 
predictor de progresión de la IAO, independientemente del grado de insuficiencia al que se asocie. Lansac y col.. proponen un algoritmo de corrección en todo reflujo excéntrico superior al trivial, sea por prolapso previo insuficientemente tratado, o una sobrecorrección restrictiva. (23) Esta situación y su adecuada interpretación representan el mayor desafío y es, al margen de la destreza técnica requerida, la representación práctica de la curva de aprendizaje. (31) La asociación de lesiones tipos 1 y 2 , y su compleja corrección, no tuvieron impacto en la progresión de IAO o en la tasa de reintervención, como fue publicado. (32)

La morbimortalidad de la serie es excelente, incluso por debajo de grandes series publicadas y consideradas en dos metaanálisis de la última década. $(16,19,33) \mathrm{La}$ reducción de los diámetros ventriculares y su contribución al mantenimiento de la FEVI, se correlacionan con la $\mathrm{CF}$ observada y la sobrevida alejada reportada a largo plazo.

Existe una clara limitación en la definición de durabilidad o estabilidad de la reparación; ¿es simple observación ecocardiográfica o la indicación real de intervención? Definida como progresión ecocardiográfica, la libertad de IAO significativa a 10 años varía entre $80 \%$ y $90 \%$, independientemente del tipo de procedimiento y el fenotipo valvular: David y ElKhoury, promotores de la técnica de reimplante, reportan $89,4 \%$ y $92 \%$ a 10 años, respectivamente $(34,15)$; Yacoub y Schafers, adeptos al remodelado $89 \%$ y $80 \%$ respectivamente, ambas series con escasa utilización de anuloplastia $(35,14)$. En nuestro medio, Escarain reporta valores similares, $84 \%$ a 8 años. (36). Esta recurrencia es bien tolerada en los pacientes con IAO previa significativa y ventrículo dilatado, y condicionan la indicación de reintervención. Por ende, si la durabilidad es representada por la tasa de reoperación, los resultados de nuestra serie son estimulantes. La libertad de intervención a 10 años raramente cae por debajo del 90\%, a excepción de patología bicúspide en los pacientes mal seleccionados (valvas restrictivas, configuración asimétrica, anuloectasia). (37)

La experiencia nos acerca a conceptos asociados al indiscutido éxito de la reparación mitral: $a$. adecuada valoración por imágenes; $b$. clasificación anatomofuncional: para cada defecto, una técnica; $c$. identificación de predictores de fallo; $d$. experiencia del cirujano; $e$. intolerancia a la regurgitación residual y $f$. curva de aprendizaje, volumen dependiente.

\section{CONCLUSIÓN}

Los procedimientos de preservación valvular aórtica son seguros y duraderos, con óptimo resultado en términos de calidad de vida, sobrevida y necesidad de reintervención, y deben ser considerados como opción en los pacientes con compromiso aneurismático de la raíz, independientemente del grado de regurgitación asociada, en especial, en aquellos que rechacen el eventual tratamiento anticoagulante o presenten contraindicación para el mismo. El reconocimiento de la anatomía funcional y su correcta tipificación ha permitido identificar aquellos pacientes no solo candidatos a la preservación valvular, sino con beneficios extendidos en el tiempo. Su correcta selección y su ejecución en centros de alto volumen y experiencia son la clave del éxito alejado.

\section{Declaración de conflictos de intereses}

Los autores declaran que no poseen conflictos de intereses.

(Véase formulario de conflicto de intereses de los autores en la web / Material suplementario).

\section{BIBLIOGRAFÍA}

1. Bentall H, De Bono A. A technique for complete replacement of the ascending aorta, Thorax 1968;23:338-9. https://doi.org/10.1136/ thx.23.4.338

2. Yacoub M, Fagan A, Stassano P, Radley-Smith R. Result of valve Conserving operations for aortic regurgitation. Circulation 1983;68;311-21

3. David TE, Feindel CM, An aortic valve-sparing operation for patients with aortic incompetence and aneurysm of the ascending aorta. J Thorac Cardiovas Surg 1992;103: 617-22. https:/doi.org/10.1016/ S0022-5223(19)34942-6

4. Lowenstein F, Guardiani P, Pieroni L, Pfister L, Carrizo E, Villegas D y col. Realidad de la cirugía cardíaca en la República Argentina. Registro CONAREC XVI. Rev Argent Cardiol 2010;78:228-37

5. Wallen T, Habertheuer A, Bavaria JE, Hughes GC, Badhwar V, Jacobs JP, et al. Ann Thorac Surg 2019;107:1307-12. https://oi. org/10.1016/j.athoracsur.2018.12.039

6. Nishimura RA, Otto CM, Bonow RO, Carabello BA, Erwin JP 3rd, Fleisher LA, et al. 2017 AHA/ACC Focused Update of the 2014 AHA/ACC Guideline for the Management of Patients With Valvular Heart Disease: A Report of the American College of Cardiology/ American Heart Association Task Force on Clinical Practice Guidelines. J Am Coll Cardiol. 2017;70:252-89. https://doi.org/10.1016/j. jacc.2017.03.011

7. Baumgartner H, Falk V, Bax JJ, De Bonis M, Hamm C, Holm PJ, et al. 2017 ESC/EACTS Guidelines for the management of valvular heart disease. Eur Heart J 2017;38:2739-91. https://doi.org/10.1093/ eurheartj/ehx391

8. le Polain de Waroux JB, Pouleur AC, Goffinet C, Vancraeynest D, Van Dyck M, Robert A, et al. Functional Anatomy of Aortic Regurgitation Accuracy, Prediction of Surgical Repairability, and Outcome Implications of Transesophageal Echocardiography. Circulation 2007;116 (suppl I):I-264-9. https://doi.org/10.1093/eurheartj/ehx391 9. Zoghbi W, Enriquez-Sarano M, Foster E, Grayburn PA, Kraft CD, Levine RA, et al. American Society of Echocardiography: Recommendations for Evaluation of the Severity of Native Valvular Regurgitation with Two-dimensional and Doppler Echocardiography A Report from the American Society of Echocardiography's Nomenclature and Standards Committee and The Task Force on Valvular Regurgitation, Developed in Conjunction with the American College of Cardiology/The American Heart Association/European Society of Cardiology Working Groups on Echocardiography. Eur J Echocardiogr2003;4:e237-e261. https://doi.org/10.1016/j.euje.2003.07.001

10. Boodhwani M, de Kerchove L, Glineur D, Poncelet A, Rubay J, Astarci P, et al. Repair-oriented classification of aortic insufficiency: impact on surgical techniques and clinical outcomes. J Thorac Cardiovasc Surg. 2009;137:286-94. https://doi.org/10.1016/j. jtcvs.2008.08.054

11. David TE. Aortic Valve Sparing in Different Aortic Valve and Aortic Root Conditions. J Am Coll Cardiol 2016;68: 654-64. https:// doi.org/10.1016/j.jacc.2016.04.062

12. David TE. Current readings: Aortic valve-sparing operations. Se- 
min Thorac Cardiovasc Surg. 2014;26:231-8. https://doi.org/10.1053/j. semtcvs.2014.10.002

13. Aphram G, Tamer S, Mastrobuoni S, El Khoury G, de Kerchove, L. Valve sparing root replacement: reimplantation of the aortic valve. Ann Cardiothoracic Surg 2019;8:415-7. https://doi.org/10.21037/ acs.2019.04.05

14. Lausberg HF, Schäfers HJ. Valve sparing aortic replacement - root remodeling. Multimed Man Cardiothorac Surg 2006;2006(1110):mm cts.2006.001982. https://doi.org/10.1510/mmcts.2006.001982

15. Mastrobuoni S, de Kerchove L, Navarra E, Watremez C, Vancraeynest D, Rubay J, et al. J Thorac Cardiovasc Surg 2019;158:14-23. https://doi.org/10.1016/j.jtcvs.2018.10.155

16. Fok M, Shaw M, Sancho E, Abello D, Bashir M. Aortic Valve Repair: A Systematic Review and Meta-analysis of Published Literature. Aort 2014;2: 10-21. http://dx.doi.org/10.12945/j.aorta.2014.14-003 17. Svensson LG, Pillai ST, Rajeswaran J, Desai MY, Griffin B, Grimm $\mathrm{R}$, et al. Long-term survival, valve durability, and reoperation for 4 aortic root procedures combined with ascending aorta replacement. J Thorac Cardiovasc Surg 2016; 151:764-71.e1-4. https://doi. org/10.1016/j.jtcvs.2015.10.113

18. Gaudino M, Lau C, Munjal M, Avgerinos D, Girardi LN. Contemporary outcomes of surgery for aortic root aneurysms: A propensitymatched comparison of valve-sparing and composite valve graft replacement. J Thorac Cardiovasc Surg 2015;150:1120-9. https://doi. org/10.1016/j.jtcvs.2015.07.015

19. Elbatarny M, Tam D, Edelman J, Rocha R, Chu M, El-Hamamsy I, et al on behalf of the Canadian Thoracic Aortic Collaborative (CTAC) Investigators, Valve-Sparing Root Replacement vs Composite Valve Grafting in Aortic Root Dilation: A Meta-Analysis. Ann Thorac Surg 2020;110:296-306 https://doi.org/10.1016/j.athoracsur.2019.11.054

20. Yokawa K, Henmi S, Nakai H, Yamanaka K, Omura A, Inoue T, et al. Mid-term outcomes of valve-sparing root reimplantation with leaflet repair. Eur J Cardiothorac Surg 2020;58:138-44. https://doi. org/10.1093/ejcts/ezaa058.doi:10.1093/ejcts/ezaa058

21. Vanoverschelde JL, van Dyck M, Gerber B, Vancraeynest D, Melchior J, de Meester C. The role of echocardiography in aortic valve repair. Ann Cardiothorac Surg 2013;2:65-7. https://doi.org/ 10.3978/j. issn.2225-319X.2012.12.04

22. Gallego P, Chaparro M, Méndez I, Castro A, Martínez-Torres MA Gómez-Domínguez R. Valoración ecocardiográfica de la anatomía funcional de la insuficiencia aórtica durante la cirugía de reparación valvular. Cir Cardiov 2014;21:181-9. https://doi.org/10.1016/j. circv.2014.02.010

23. Berrebi A, Monin JL, Lansac E. Systematic echocardiographic assessment of aortic regurgitation - $i$ what should the surgeon know for aortic valve repair? Ann Cardiothorac Surg 2019;8:331-41. https:// doi.org/10.21037/acs.2019.05.15

24. Karliova I, Schneider U, Ehrlich T, Schäfers HJ. Results of Pericardial Patches in Tricuspid and Bicuspid Aortic Cusp Repair.
Ann Thorac Surg 2020; 109:728-35. https://doi.org/10.1016/j.athoracsur.2019.07.025

25. Schäfers HJ, Langer F, Glombitza P, Kunihara T, Fries R, Aicher D. Aortic valve reconstruction in myxomatous degeneration of aortic valves: Are fenestrations a risk factor for repair failure? J Thorac Cardiovasc Surg 2010;139:660-4. https://doi.org/10.1016/j.jtcvs.2009.06.025 26. Nash PJ, Vitvitsky E, Li J, Cosgrove DM, Pettersson G, Grimm RA. Feasibility of valve repair for regurgitant bicuspid aortic valves-an echocardiographic study. Ann Thorac Surg 2005;79:1473-9. https:// doi.org/10.1016/j.athoracsur.2004.09.053

27. David TE. Remodeling the Aortic Root and Preservation of the Native Aortic Valve. Operative Techn Cardiac Thorac Surg 1996;1:4456. https://doi.org/10.1016/S1085-5637(07)70080-3

28. Schneider U, Hofmann C, Aicher D, Takahashi H, Miura Y, Schäfers HJ. Suture annuloplasty significantly improves the durability of bicuspid aortic valve repair. Ann Thorac Surg 2017;103:504-10. https://doi.org/10.1016/j.athoracsur.2016.06.072

29. Lansac E, Di Centa I, Bonnet N, Leprince P, Rama A, Acar C, et al. Aortic prosthetic ring annuloplasty: a useful adjunct to a standardized aortic valve-sparing procedure? Eur J Cardiothorac Surg 2006;29: 537-44. https://doi.org/10.1016/j.ejcts.2005.12.055

30. Lansac E, Di Centa I, Vojacek J, Leprince P, Rama A, Acar C, et al. Valve sparing root replacement: the remodeling technique with external ring annuloplasty. Ann Cardiothorac Surg 2013;2:117-23. https://doi.org/10.3978/j.issn.2225-319X.2013.01.15

31. Malas T, Saczkowski R, Sohmer, Ruel M, Mesana T, de Kerchove L, et al. Is Aortic Valve Repair Reproducible? Analysis of the Learning Curve for Aortic Valve Repair. Can J Cardiol 2015 31:1497.e15-1497. e22. https://doi.org/ 10.1016/j.cjca.2015.05.016

32. Langer F. Graeter T. Nikoloudakis N. et al. Valve-preserving aortic replacement: does the additional repair of leaflet prolapse adversely affect the results?.J Thorac Cardiovasc Surg. 2001;122: 270-7. https:// doi.org/10.1067/mtc.2001.114635

33. Harky A, Antoniou A, Howard C, Rimmer L, Ahmad MU, Bashir $\mathrm{M}$. Valve sparing aortic root surgery: from revolution to evolution? J Vis Surg 2019;5:14. https://doi.org/10.21037/jovs.2019.01.11

34. David TE, David CM, Feindel CM, Manlhiot C. Reimplantation of the Aortic Valve at 20 years. J Thorac Cardiovasc Surg 2017;153:232-8 http://dx.doi.org/10.1016/j.jtcvs.2016.10.081

35. Yacoub MH, Gehle P, Chandrasekaran V, et al. Late results of a valve-preserving operation in patients with aneurysms of the ascending aorta and root. J Thorac Cardiovasc Surg 1998;115:1080-90. https://doi.org/10.1016/S0022-5223(98)70408-8

36. Escaraín M, Duczinsky G, Bozovich E, Favaloro R. Cirugía de preservación de la válvula aórtica con técnica de reimplante, Rev Argent Cardiol 2014;82:303-9. https://doi.org/10.7775/rac.es.v82.i4.2276 37. Ehrlich T, de Kerchove L, Vojacek J, Boodhwani M, El-Hamamsy I, De Paulis R, et al. State-of-the art bicuspid aortic valve repair in 2020. Prog Cardiovasc Dis 2020;63:457-64. https://doi.org/10.1016/j. pcad.2020.04.010 


\section{MATERIAL SUPLEMENTARIO}

Apéndice 1. Definiciones de morbilidad

Mediastinitis: infección de tejidos profundos con signos clínicos o rescate microbiológico, independiente de la estabilidad esternal. CDC/NHSN Surveillance Definitions for Specific Types of Infections, Jannuary 2020

Insuficiencia renal: por criterios RIFLE, tipo 2 daño, elevación de creatinina basal en 2-3 veces el valor basal o diuresis <0,5ml/kg/h en 12 horas. Bellomo R, Ronco C, Kellum JA, Mehta RL, Palevsky P. Acute renal failuredefinition, outcome measures, animal models, fluid therapy and information technology needs: the Second International Consensus Conference of the Acute Dialysis Quality Initiative (ADQI) Group. Crit Care 2004;8:R204-12.

AIT, accidente isquémico transitorio de duración menor a 24 horas. ACV, accidente cerebrovascular, con duración superior a 24 horas, o con secuela clínica o por imágenes. Lansky AJ, Messé SR, Brickman AM, Dwyer M, Bart van der Worp H, Lazar RM, et al. Proposed Standardized Neurological Endpoints for Cardiovascular Clinical Trials. J Am Coll Cardiol 2017;69:679-91.

Uso de inotrópicos, como resultado de bajo gasto cardíaco clínico o por medición de Swan Ganz posoperatorio.

ARM prolongada, superior a 24 horas posoperatorias.

Sangrado médico, superior a $800 \mathrm{~m}$ en 12 horas, con transfusión de ms de 2 unidades de GRS, plaquetas o crioprecipitados. Cornelius Dyke, Solomon Aronson, Wulf Dietrich, Axel Hofmann, Keyvan Karkouti, MarcelLevi, Gavin J.Murphy, Frank W.Sellke, et al. Universal definition of perioperative bleeding in adult cardiac surgery., J Thorac Cardiovasc Surg2014; 147:1458-63.https://doi.org/10.1016/j.jtcvs.2013.10.070 